Article

\title{
Comparing Immune Responses to Inactivated Vaccines against SARS-CoV-2 between People Living with HIV and HIV-Negative Individuals: A Cross-Sectional Study in China
}

\author{
Xiaojie Huang ${ }^{1,+}$, Ying Yan ${ }^{2,+}$, Bin Su ${ }^{1,+} \mathbb{D}$, Dong Xiao ${ }^{3,+}$, Maohe Yu ${ }^{4}$, Xia Jin ${ }^{5}$, Junyi Duan ${ }^{1}$, Xiangjun Zhang ${ }^{6} \mathbb{D}$, \\ Shimin Zheng ${ }^{7}$, Yuan Fang ${ }^{8} \mathbb{D}$, Tong Zhang ${ }^{1}$, Weiming Tang ${ }^{9, *}$, Lunan Wang ${ }^{2, *}$, Zixin Wang ${ }^{10, *} \mathbb{D}$ \\ and Junjie $\mathrm{Xu}{ }^{11, *}$
}

check for

updates

Citation: Huang, X.; Yan, Y.; Su, B.; Xiao, D.; Yu, M.; Jin, X.; Duan, J.; Zhang, X.; Zheng, S.; Fang, Y.; et al. Comparing Immune Responses to Inactivated Vaccines against SARS-CoV-2 between People Living with HIV and HIV-Negative Individuals: A Cross-Sectional Study in China. Viruses 2022, 14, 277. https://doi.org/10.3390/v14020277 Academic Editor: Ester Ballana Guix Received: 28 December 2021 Accepted: 26 January 2022 Published: 28 January 2022 Publisher's Note: MDPI stays neutral with regard to jurisdictional claims in published maps and institutional affiliations.

Copyright: (C) 2022 by the authors. Licensee MDPI, Basel, Switzerland. This article is an open access article distributed under the terms and conditions of the Creative Commons Attribution (CC BY) license (https:// creativecommons.org/licenses/by/ $4.0 /)$.
1 Clinical and Research Center for Infectious Diseases, Beijing Youan Hospital, Capital Medical University, Beijing 100069, China; huangxiaojie78@ccmu.edu.cn (X.H.); binsu@ccmu.edu.cn (B.S.); youanduanyi@163.com (J.D.); zt_doc@ccmu.edu.cn (T.Z.)

2 National Center for Clinical Laboratories, Beijing Hospital, National Center of Gerontology, Institute of Geriatric Medicine, Chinese Academy of Medical Sciences, Beijing 100730, China; yyan511@nccl.org.cn

3 Rainbow Clinic of Beijing Jingcheng Skin Hospital, Beijing 100101, China; hivct@me.com

4 Department of AIDS/STD Control and Prevention, Tianjin Center for Disease Control and Prevention, Tianjin 300011, China; yumaohe@tj.gov.cn

5 AIDS Healthcare Foundation (AHF), Beijing 100088, China; jinxiajiangsu@126.com

6 Department of Public Health, The University of Tennessee, Knoxville, TN 37996, USA; xzhan140@utk.edu

7 Department of Biostatistics and Epidemiology, East Tennessee State University, Johnson City, TN 70300, USA; zhengs@etsu.edu

8 Department of Health and Physical Education, The Education University of Hong Kong, Hong Kong 200092, China; lunajoef@gmail.com

9 University of North Carolina Project-China, Guangzhou 510095, China

10 JC School of Public Health and Primary Care, Faculty of Medicine, The Chinese University of Hong Kong, Hong Kong 666888, China

11 Clinical Research Academy, Peking University Shenzhen Hospital, Peking University, Shenzhen 518036, China

* Correspondence: Weiming_tang@med.unc.edu (W.T.); lnwang@nccl.org.cn (L.W.); wangzx@cuhk.edu.hk (Z.W.); xjjcmu@163.com (J.X.)

+ Authors contributed equally as first authors.

Abstract: This study compared the immunogenicity of inactivated SARS-CoV-2 vaccines between people living with HIV (PLWH) and HIV-negative individuals. We recruited 120 PLWH and 53 HIVnegative individuals aged $18-59$ years who had received an inactivated SARS-CoV-2 vaccine in two Chinese cities between April and June 2021. Blood samples were tested for immunogenicity of the inactivated SARS-CoV-2 vaccines. The prevalence and severity of adverse events associated with SARS-CoV-2 vaccines were similar between PLWH and HIV-negative individuals. The seropositivity of neutralizing activity against authentic SARS-CoV-2, of the total amount of antibody (total antibody) and of S-IgG were $71.3 \%, 81.9 \%$, and 92.6\%, respectively, among fully vaccinated PLWH. Among all participants, PLWH had lower neutralizing activity, total antibody, S-IgG, and T-cell-specific immune response levels, compared to HIV-negative individuals, after controlling for types of vaccine, time interval between first and second dose, time after receiving the second dose, and sociodemographic factors. PLWH with a longer interval since HIV diagnosis, who received their second dose 15-28 days prior to study commencement, and who had an interval of $\geq 21$ days between first and second dose had higher neutralizing activity levels. The immunogenicity of the inactivated SARS-CoV-2 vaccines was lower among PLWH as compared to HIV-negative individuals. Vaccination guideline specific for PLWH should be developed.

Keywords: people living with HIV; inactivated SARS-CoV-2 vaccines; self-reported adverse events; neutralizing activity responses against authentic SARS-CoV-2; total antibody specific to SARS-CoV-2; SARS-CoV-2 IgG antibody; antigen-specific T-cell immune response 


\section{Introduction}

Globally, about 38 million people are living with HIV [1]. Antiretroviral therapy (ART) has been shown to suppress viral replication, restore CD4+ T-cell counts, rebuild immune function, and decrease morbidity and mortality among people living with HIV (PLWH) [2,3]. However, CD4+ T-cell recovery is incomplete despite viral suppression in some PLWH [4]. There is conflicting evidence as to whether PLWH have an increased risk of SARS-CoV-2 infection [5,6]. Several systematic reviews and non-systematic reviews found comparable mortality and morbidity of SARS-CoV-2 between PLWH and HIV-negative individuals [7-11]. SARS-CoV-2 vaccination is important for all patients with potentially decreased immune responses (e.g., patients with autoimmune diseases or who are receiving immunosuppressive agents, and PLWH) [12]. Both international health authorities and Chinese national guidelines recommend SARS-CoV-2 vaccination for PLWH regardless of their immune status [13-15].

PLWH are considered a priority group for vaccination in many countries [15]. However, there are concerns that PLWH might have a suboptimal response to SARS-CoV-2 vaccination. More importantly, less than 3\% of the participants in the reported SARS-CoV-2 vaccine efficacy trials are PLWH, and the data for vaccine safety and immune response are insufficient [16-19]. Some studies have compared the safety and immunogenicity of mRNA (Pfizer BNT162b2 and Moderna mRNA-1273) or adenovirus vector (Oxford/AstraZeneca AZD1222) SARS-CoV-2 vaccines between HIV-negative individuals and PLWH who have viral suppression and high CD4+ T-cell levels (median around 700) [20-24]. These studies showed that SARS-CoV-2 vaccines were safe for PLWH, and that there was no betweengroup difference in adverse events [20-24].

There are two inactivated SARS-CoV-2 vaccines manufactured by Chinese companies that are approved for emergency use by the World Health Organization (WHO) (Sinopharm and Sinovac CoronaVac) [25,26]. More than three billion doses of these vaccines have been supplied to more than 40 countries [27]. No study has compared PLWH and HIV-negative individuals when it comes to the immunogenicity and safety of the inactivated SARS-CoV-2 vaccines. Such evidence is important to address COVID-19 vaccine hesitancy among PLWH and to implement booster doses for this group [28]. Previous findings on mRNA/adenovirus vector vaccines might not be applicable to PLWH receiving inactivated SARS-CoV-2 vaccines [20-24]. Moreover, it is unclear whether PLWH with lower CD4+ T-cell counts and with detectable HIV viral loads would have similar immunogenicity as HIV-negative individuals, as these PLWH were excluded by the aforementioned studies [20-24]. Furthermore, given the relatively short follow-up period in previous studies, there is no consensus about the long-term immunogenicity of SARS-CoV-2 vaccines among PLWH [20-24].

This study aims to address these knowledge gaps by comparing the immunogenicity and adverse events associated with these vaccines between PLWH and HIV-negative individuals after vaccination. This study also investigated factors correlated with levels of neutralizing activity against authentic SARS-CoV-2, the total antibody specific to SARSCoV-2, SARS-CoV-2 IgG antibody against the receptor-binding domain (RBD) of the spike protein (S-IgG), and antigen-specific T-cell immune response among PLWH.

\section{Materials and Methods}

\subsection{Study Design}

This cross-sectional study was conducted in two Chinese metropolitan cities (Beijing and Tianjin) between April and June 2021. Participants included PLWH and HIV-negative individuals who had received at least one dose of inactivated SARS-CoV-2 vaccine.

\subsection{Participants}

The inclusion criteria for PLWH included the following: (1) aged 18-59 years; (2) willing to participate in the study activities, including survey and blood sample collection, and relevant laboratory testing; (3) having received at least one dose of inactivated SARS- 
CoV-2 vaccine (Sinovac CoronaVac or Sinopharm); and (4) having received an HIV diagnosis confirmed by HIV-1/2 Western blot assay. Exclusion criteria included the following: (1) presence of severe hearing loss, impaired vision, or intellectual disability observed by the interviewers; or (2) a history of SARS-CoV-2 infection, major psychiatric illness (schizophrenia or bipolar disorder) or neurocognitive impairment based on clinician's assessment of their medical records. HIV-negative individuals shared the first three inclusion criteria and both exclusion criteria with PLWH. HIV serostatus was confirmed by Abbott ARCHITECT HIV Ag/Ab Combo assay.

\subsection{Recruitment and Data Collection}

Recruitment for PLWH was facilitated by two community-based organizations (CBOs), one in each city. These two CBOs have provided services to PLWH and HIV high-risk populations and worked closely with HIV clinical service providers. WeChat is the most commonly used social media application for the CBOs to communicate with PLWH clients. $\mathrm{CBO}$ staff posted the study recruitment information in the WeChat public accounts of their organizations. Interested PLWH contacted CBO staff through private WeChat messages, phone calls, or messages via other instant messaging applications. CBO staff screened participants' eligibility, briefed them about the study purpose and procedures, assured them that identifiable information would be kept confidential, and informed them that their refusal to participate would have no consequences. The recruitment of HIV-negative individuals was conducted in community hospitals. The hospital staff identified vaccinated individuals based their patient records and contacted them by telephone to invite them to participate.

PLWH and HIV-negative individuals interested in joining the study were invited to visit one of two clinics, based in each city. Whilst prospective participants were on site, project staff obtained their written informed consent. All participants also completed a 10-min self-administered questionnaire whilst on site.

\subsection{Blood Sample Collection and Laboratory Procedures}

After completion of the survey, trained nurses collected two lithium heparin anticoagulated vacuum blood collection tubes (BD) of whole blood $(10 \mathrm{~mL})$, two EDTA anticoagulated vacuum blood collection tubes (BD) of whole blood $(10 \mathrm{~mL})$, and one SST blood collection tube of whole blood $(5 \mathrm{~mL})$. One tube of lithium heparin salt anticoagulated whole blood and one tube of EDTA anticoagulated whole blood were placed at room temperature. They were assayed for T-cell-specific immune response within $8 \mathrm{~h}$ and for CD4+ T-cell count within $48 \mathrm{~h}$, respectively. The other three tubes of whole blood were centrifuged at 1300 relative centrifugal force (RCF) for $10 \mathrm{~min}$, and the upper plasma/serum layers were transferred into lyophilized tubes of no less than $1.2 \mathrm{~mL}$ each, then stored at $-20{ }^{\circ} \mathrm{C}$ for the detection of SARS-CoV-2 combined antibody and neutralizing activity, as well as HIV viral load.

SARS-CoV-2 neutralizing activity measurement. The neutralizing activity to authentic SARS-CoV-2 (virus strain SARS-CoV-2/human/CHN/CN1/2020, GenBank number MT407649.1) was quantified using a micro cytopathogenic effect (CPE) inhibition assay with a minimum four-fold dilution, as reported before [29]. This neutralization assay is considered the gold standard measurement of neutralizing activity. The positive geometric mean titer (GMT) of the neutralizing activity to authentic SARS-CoV-2 was 8.

SARS-CoV-2 antibody combined testing. All samples were tested for SARS-CoV-2 specific total antibody and S-IgG antibodies using Chemiluminescence assay (CLIA) kits (Beijing Wantai Biological Pharmacy Enterprise Co., Ltd., Beijing, China). The total antibody was detected by double-antigen sandwich-based method, which could detect the total amount of SARS-CoV-2-specific IgG, IgM, and IgA. S-IgG was detected by indirect method. The positive cut-off for the abovementioned tests was 1.0.

T-cell-specific immune response. The T-cell-specific immune response was tested using the IFN- $\gamma$ release assay (Beijing Wantai Biological Pharmacy Enterprise Co., Ltd., 
Beijing, China). Briefly, $1.5 \mathrm{~mL}$ of heparin, blood was distributed into a test tube containing specific SARS-CoV-2 S-antigen ( $\mathrm{T}$ tube), a negative control tube ( $\mathrm{N}$ tube), and a positive control tube (P tube) within $8 \mathrm{~h}$. The tubes were inverted and mixed 5 times, then incubated in $37^{\circ} \mathrm{C}$ for $20-24 \mathrm{~h}$. Then, the plasma was collected after centrifuging at $3000 \mathrm{RCF}$ for $10 \mathrm{~min}$ and assessed for IFN- $\gamma$ level. If the level of $\mathrm{T}$ tube minus $\mathrm{N}$ tube resulted in a value greater than $30 \mathrm{pg} / \mathrm{mL}$ the result was considered positive.

HIV viral load. Viral load of PLWH was tested using HIV quantitative assay (Zhuhai Livzon Diagnostics Inc., Zhuhai, China). The limit of quantitation (LOQ) of this assay was 60 copies/mL.

CD4+ cell count measurement. The assay was performed using flow cytometry testing methods (BD Biosciences, San Jose, CA, USA) in accordance with the China National Guideline for Detection of HIV/AIDS (version 2020) [30].

Background characteristics of the participants. All participants reported age, gender, and presence of chronic conditions (i.e., hypertension, hyperlipidemia, cancer, chronic cardiovascular, lung, liver, or kidney diseases, and diabetes mellitus). Characteristics related to HIV infection and SARS-CoV-2 vaccination were extracted from medical records.

Adverse events related to SARS-CoV-2 vaccination. A checklist was used to assess local adverse events (pain, redness, itching, swelling, induration, and skin rashes in the arm where the shot was given) and systematic adverse events (fatigue, malaise, headache, dizziness, lethargy, joint pain or muscle ache, fever, nausea, vomiting, diarrhea, and others) within one month after receiving a SARS-CoV-2 vaccine. Participants rated the severity of the aforementioned adverse events $(1=$ very mild, $2=$ mild, $3=$ moderate, $4=$ severe, and 5 = very severe).

\subsection{Sample Size Planning}

Previous studies showed that the positive rate for SARS-CoV-2 neutralizing activity was about $90 \%$ among HIV-negative individuals who received inactivated SARS-CoV-2 vaccines [29]. There were no data on seropositivity for SARS-CoV-2 neutralizing activity among PLWH who received inactivated vaccines. Previous studies showed that the seroconversion rate of PLWH after inoculation with the hepatitis B vaccine ranged from 34\% to $88 \%$ [31]. Therefore, we assumed that $70 \%$ of vaccinated PLWH would be positive for SARS-CoV-2 neutralizing activity. Using an allocation ratio of 2:1, a total of 102 PLWH and $51 \mathrm{HIV}$-negative individuals were required to detect a minimum between-group difference of $20 \%(90 \%$ versus $70 \%)$ in the positivity rate of SARS-CoV-2 neutralizing activity $(\alpha=0.05$, $\beta=0.10)$.

\subsection{Statistical Analysis}

Chi-square tests were used to inspect the difference in background characteristics and adverse events related to SARS-CoV-2 vaccination between PLWH and HIV-negative individuals. Between-group differences in immunogenicity indicator levels (total antibody, neutralizing activity, S-IgG, and T-cell-specific immune response) were tested using MannWhitney tests. We log transformed the immunogenicity indicator levels using a base of 10 to normalize the data. Multivariable linear regression models were performed to test the between-group differences in these indicators, after controlling for all background characteristics with $p<0.05$ in between-group comparisons. Adjusted coefficients (B) were obtained. Moreover, same comparisons were performed between different subgroups of PLWH and HIV-negative individuals. Similar analyses on seropositivity for these immunogenicity indicators were also performed. Among PLWH, linear regression models were used to inspect factors that were correlated with immunogenicity indicator levels. In addition, correlations between CD4+ T-cell count/viral load and years since diagnosis were investigated. SPSS version 26.0 was used in all analyses, with two-tailed $p<0.05$ considered statistically significant. 


\subsection{Ethics Statement}

Written informed consent was obtained from all participants before their study participation in accordance with the Declaration of Helsinki. The Institutional Review Boards of Changzhi Medical College (RT2021002) and Beijing Youan Hospital Research Ethics Committee (No. 2021-031) approved this study.

\section{Results}

\subsection{Profiles of the Participants}

A total of 519 and 316 PLWH in Beijing and Tianjin were approached. A total of 130 and 24 from each city, respectively, were screened as eligible, and $110(84.6 \%)$ and 19 (79\%), respectively, completed the study. During the same period, 61 vaccinated HIV-negative individuals were approached. Eight (13.1\%) refused to participate mainly due to logistical reasons, and 53 (86.9\%) completed the study procedures. Most PLWH had received HIV diagnoses over one year ago (86\%), and most were on ART $(97.7 \%)$. Over half of them had an undetectable viral load (58.1\%). The median HIV viral load and CD4+ T-cell counts were 42 (IQR: 0, 117) and 630.5 (IQR: 499.5, 848.8), respectively (Table 1).

Table 1. Background characteristics of HIV-negative individuals and people living with HIV (PLWH) who had received at least one dose of SARS-CoV-2 vaccine.

\begin{tabular}{|c|c|c|c|}
\hline & $\begin{array}{l}\text { People Living with HIV } \\
\text { (n = 129) }\end{array}$ & $\begin{array}{l}\text { HIV-Negative Individuals } \\
\qquad(\mathrm{n}=53)\end{array}$ & $p$ Values \\
\hline \multicolumn{4}{|l|}{ Socio-Demographics } \\
\hline \multicolumn{4}{|l|}{ Age (years), n (\%) } \\
\hline $18-29$ & $39(30.2)$ & $14(26.4)$ & \\
\hline $30-39$ & $65(50.4)$ & $19(35.8)$ & \\
\hline $40-49$ & $20(15.5)$ & $11(20.8)$ & \\
\hline $50-59$ & $5(3.9)$ & $9(17.0)$ & 0.01 \\
\hline Median (IQR), range & $\begin{array}{c}34(28,38) \\
(20-58)\end{array}$ & $\begin{array}{c}34(29,47) \\
(22-56)\end{array}$ & 0.15 \\
\hline \multicolumn{4}{|l|}{ Gender, n (\%) } \\
\hline Male & $128(99.2)$ & $40(75.5)$ & \\
\hline Female & $1(0.8)$ & $13(24.5)$ & $<0.001$ \\
\hline \multicolumn{4}{|c|}{ Presence of chronic conditions other than HIV / AIDS } \\
\hline No & $102(79.1)$ & $53(100.0)$ & \\
\hline Yes & $27(20.9)$ & $0(0.0)$ & $<0.001$ \\
\hline \multicolumn{4}{|c|}{ Characteristics related to $\mathrm{HIV}$ infection } \\
\hline$\leq 1$ & $18(14.0)$ & N.A & N.A. \\
\hline $2-5$ & $55(42.6)$ & N.A & N.A. \\
\hline $6-10$ & $35(27.1)$ & N.A & N.A. \\
\hline$>10$ & $21(16.3)$ & N.A & N.A. \\
\hline \multicolumn{4}{|l|}{ Viral load (cp/mL), n (\%) } \\
\hline Undetectable $(\leq 60)$ & $75(58.1)$ & N.A. & N.A. \\
\hline $61-200$ & $33(25.6)$ & N.A. & N.A. \\
\hline$>200$ & $21(16.3)$ & N.A. & N.A. \\
\hline Median (IQR), range & $\begin{array}{l}41(0,117) \\
(0,50397)\end{array}$ & N.A. & N.A. \\
\hline \multicolumn{4}{|c|}{ CD4+ T-cell count (cells $/ \mu \mathrm{L})$} \\
\hline$<500$ & $32(24.8)$ & N.A. & N.A. \\
\hline $500-1000$ & $81(62.8)$ & N.A. & N.A. \\
\hline$>1000$ & $16(12.4)$ & N.A. & N.A. \\
\hline Median (IQR), range & $\begin{array}{l}630.5(499.5,848.8) \\
\quad(78,2650.35)\end{array}$ & N.A. & N.A. \\
\hline
\end{tabular}


Table 1. Cont.

\begin{tabular}{|c|c|c|c|}
\hline & $\begin{array}{l}\text { People Living with HIV } \\
\text { (n = 129) }\end{array}$ & $\begin{array}{l}\text { HIV-Negative Individuals } \\
\qquad(\mathrm{n}=53)\end{array}$ & $p$ Values \\
\hline \multicolumn{4}{|l|}{ ART regimens } \\
\hline $\mathrm{TDF}+3 \mathrm{TC}+\mathrm{EFV}$ & $60(52.7)$ & N.A. & N.A. \\
\hline $\mathrm{TDF}+3 \mathrm{TC}+\mathrm{LPV} / \mathrm{r}$ & $5(3.9)$ & N.A. & N.A. \\
\hline $\mathrm{AZT}+3 \mathrm{TC}+\mathrm{LPV} / \mathrm{r}$ & $3(2.3)$ & N.A. & N.A. \\
\hline $\mathrm{AZT}+3 \mathrm{TC}+\mathrm{NVP}$ & $2(1.6)$ & N.A. & N.A. \\
\hline $\mathrm{AZT}+3 \mathrm{TC}+\mathrm{EFV}$ & $8(6.2)$ & N.A. & N.A. \\
\hline Others & $40(31.0)$ & N.A. & N.A. \\
\hline Not on ART & $3(2.3)$ & N.A. & N.A. \\
\hline \multicolumn{4}{|l|}{$\begin{array}{c}\text { Information related to SARS-CoV-2 vaccination } \\
\text { SARS-CoV-2 vaccination status }\end{array}$} \\
\hline Partially vaccinated & $35(27.1)$ & $2(3.8)$ & \\
\hline 0-14 days since fully vaccinated & $15(11.6)$ & $8(15.1)$ & \\
\hline $15-28$ days since fully vaccinated & $38(29.5)$ & $13(25.5)$ & \\
\hline $29-56$ days since fully vaccinated & $26(20.2)$ & $21(39.6)$ & \\
\hline 57-84 days since fully vaccinated & $12(9.3)$ & $3(5.7)$ & \\
\hline$>84$ days since fully vaccinated & $3(2.3)$ & $8(15.1)$ & $<0.001$ \\
\hline \multicolumn{4}{|l|}{ Type of SARS-CoV-2 vaccine } \\
\hline Sinopharm & $58(45.0)$ & $37(69.8)$ & \\
\hline Sinovac CoronaVac & $71(55.0)$ & $16(30.2)$ & $<0.001$ \\
\hline $\begin{array}{l}\text { Time interval between the first and second dose } \\
\text { (among those who were fully vaccinated) }\end{array}$ & $\mathrm{n}=94$ & $\mathrm{n}=51$ & \\
\hline$<21$ days & $20(21.3)$ & $3(5.7)$ & \\
\hline $21-28$ days & $58(61.7)$ & $40(75.5)$ & \\
\hline$>28$ days & $16(17.0)$ & $10(18.9)$ & 0.043 \\
\hline Median (IQR), range & $\begin{array}{c}21(21,27) \\
(14-59)\end{array}$ & $\begin{array}{l}27(21,28) \\
(14-83)\end{array}$ & 0.002 \\
\hline
\end{tabular}

N.A.: not applicable.

As compared to HIV-negative individuals, fewer PLWH were 50-59 years old (3.9\% versus $17.0 \%, p=0.01)$ and female $(0.8 \%$ versus $24.5 \%, p<0.001)$. More PLWH had chronic conditions $(20.9 \%$ versus $0 \%, p<0.001)$, received Sinovac CoronaVac $(55.0 \%$ versus $30.2 \%, p<0.001)$, and had received only the first dose $(27.1 \%$ versus $3.8 \%, p<0.001)$. No participants had received more than one type of vaccine. Among those who completed both doses, the time interval between the first and second dose was shorter among PLWH than among HIV-negative individuals (median: 21 versus 27 days, $p<0.001$ ) (Table 1 ). These background characteristics were controlled when comparing immunogenicity indicator levels between PLWH and HIV-negative individuals.

\subsection{SARS-CoV-2 Vaccination Adverse Events}

Among the participants, $45.0 \%$ of PLWH and $54.7 \%$ of HIV-negative individuals reported experiencing specific local or systematic adverse events. After controlling for significant background characteristics (i.e., age group, gender, presence of chronic conditions other than HIV, type of vaccine, time interval between first and second dose, and time after receiving the second dose), there was no between-group difference in prevalence of adverse events (AOR: 0.77,95\% CI: 0.31, 1.95, $p=0.19$ ). Most of the reported adverse events were very mild/mild (41-100\% among PLWH and 62.2-100\% among HIV-negative individuals). There was no between-group difference in the severity of these adverse events $(p=0.13-0.77)$ (Table 2). 
Table 2. Comparing self-reported local and systematic adverse events related to SARS-CoV-2 vaccination among People living with HIV (PLWH) and HIV-negative individuals.

\begin{tabular}{|c|c|c|c|}
\hline & $\begin{array}{l}\text { People Living with HIV } \\
\text { (n = 129) }\end{array}$ & $\begin{array}{l}\text { HIV-Negative Individuals } \\
\qquad(\mathrm{n}=53)\end{array}$ & $p$ Values \\
\hline & $\mathrm{n}(\%)$ & $\mathrm{n}(\%)$ & \\
\hline \multicolumn{4}{|l|}{ Local Adverse Events } \\
\hline \multicolumn{4}{|l|}{ Pain } \\
\hline None & $87(67.4)$ & $31(58.5)$ & \\
\hline Very mild & $15(11.6)$ & $4(7.5)$ & \\
\hline Mild & $16(12.4)$ & $11(20.8)$ & \\
\hline Moderate & $11(8.5)$ & $7(13.2)$ & \\
\hline Severe & $0(0.0)$ & $0(0.0)$ & 0.30 \\
\hline Any of above & $42(32.6)$ & $22(41.5)$ & 0.25 \\
\hline \multicolumn{4}{|l|}{ Redness, itch, swelling, induration, and/or skin rash } \\
\hline None & $124(96.1)$ & $50(94.3)$ & \\
\hline Very mild & $0(0.0)$ & $1(1.9)$ & \\
\hline Mild & $2(1.6)$ & $2(3.8)$ & \\
\hline Moderate & $3(2.3)$ & $0(0.0)$ & \\
\hline Severe & $0(0.0)$ & $0(0.0)$ & 0.21 \\
\hline Any of above & $5(3.9)$ & $3(5.7)$ & 0.59 \\
\hline \multicolumn{4}{|l|}{ Systematic adverse events } \\
\hline \multicolumn{4}{|l|}{ Fatigue, malaise, headache, dizziness, and/or lethargy } \\
\hline None & $107(82.9)$ & $43(81.1)$ & \\
\hline Very mild & $5(3.9)$ & $3(5.7)$ & \\
\hline Mild & $11(8.5)$ & $4(7.5)$ & \\
\hline Moderate & $5(3.9)$ & $2(3.8)$ & \\
\hline Severe & $1(0.8)$ & $1(1.9)$ & 0.94 \\
\hline Any of above & $22(17.1)$ & $10(18.9)$ & 0.77 \\
\hline \multicolumn{4}{|l|}{ Joint pain and/or muscle ache } \\
\hline None & $119(92.2)$ & $45(84.9)$ & \\
\hline Very mild & $4(3.1)$ & $1(1.9)$ & \\
\hline Mild & $3(2.3)$ & $4(7.5)$ & \\
\hline Moderate & $3(2.3)$ & $3(5.7)$ & \\
\hline Severe & $0(0.0)$ & $0(0.0)$ & 0.23 \\
\hline Any of above & $10(7.8)$ & $8(15.1)$ & 0.13 \\
\hline \multicolumn{4}{|l|}{ Fever } \\
\hline None & $122(94.6)$ & $52(98.1)$ & \\
\hline Very mild & $2(1.6)$ & $0(0.0)$ & \\
\hline Mild & $4(3.1)$ & $1(1.9)$ & \\
\hline Moderate & $1(0.8)$ & $0(0.0)$ & \\
\hline Severe & $0(0.0)$ & $0(0.0)$ & 0.69 \\
\hline Any of above & $7(5.4)$ & $1(1.9)$ & 0.27 \\
\hline \multicolumn{4}{|l|}{ Nausea, vomit, and/or diarrhea } \\
\hline None & $129(100.0)$ & $52(98.1)$ & \\
\hline Very mild & $0(0.0)$ & $0(0.0)$ & \\
\hline Mild & $0(0.0)$ & $1(1.9)$ & \\
\hline Moderate & $0(0.0)$ & $0(0.0)$ & \\
\hline Severe & $0(0.0)$ & $0(0.0)$ & 0.12 \\
\hline Any of above & $0(0.0)$ & $1(1.9)$ & 0.29 \\
\hline \multicolumn{4}{|l|}{ Other systematic side-effects } \\
\hline None & $127(98.4)$ & $53(100.0)$ & \\
\hline Very mild & $2(1.6)$ & $0(0.0)$ & \\
\hline Mild & $0(0.0)$ & $0(0.0)$ & \\
\hline Moderate & $0(0.0)$ & $0(0.0)$ & \\
\hline Severe & $0(0.0)$ & $0(0.0)$ & 0.36 \\
\hline Any of above & $2(1.6)$ & $0(0.0)$ & 0.50 \\
\hline Any local and/or systematic adverse events & $58(45.0)$ & $29(54.7)$ & 0.23 \\
\hline
\end{tabular}




\subsection{Immunogenicity Indicator Levels}

The seropositivity of neutralizing activity, the total antibody, and the S-IgG values were $71.3,81.9$ and $92.6 \%$, respectively, among fully vaccinated PLWH. The prevalence of seropositivity of all four immunogenicity indicators was lower among fully vaccinated PLWH than among fully vaccinated HIV-negative individuals, with the exception of neutralizing activity. (Tables 3 and S1).

When compared to HIV-negative individuals, PLWH had significantly lower levels of neutralizing activity (adjusted B: $-0.18, p=0.049$ ), total antibody (adjusted B: -0.80 , $p<0.001$ ), S-IgG (adjusted B: $-0.31, p=0.002$ ), and T-cell-specific immune response (adjusted B: $-0.64, p=0.002$ ). Subgroup analyses showed that all subgroups of PLWH had significantly lower neutralizing activity than HIV-negative individuals, with the exception of the subgroup of PLWH with CD4+ T-cell counts $\geq 500$ and with undetectable viral loads (adjusted B: $-0.23, p=0.16$ ). In addition, PLWH had significantly lower levels of total antibody, S-IgG, and T-cell-specific immune response, regardless of CD4+ T-cell counts or levels of HIV viral suppression. Neutralizing activity levels among fully vaccinated PLWH were not lower than those among fully vaccinated HIV-negative individuals (adjusted B: $-0.15, p=0.13$ ) (Tables 4 and 5).

\subsection{Factors Associated with Immunogenicity Indicator Levels among PLWH}

A longer time since HIV diagnosis was associated with higher neutralizing activity and total antibody levels (2-5 years: adjusted B: 0.71 and 0.27 ; reference: $\leq 1$ year). As compared to partially vaccinated participants, PLWH who had received their second dose 15-28 days previously had higher neutralizing activity levels (adjusted B: 0.35), while those who had received it 15-56 days previously had higher levels of total antibody (adjusted B: 1.03), S-IgG (adjusted B: 0.73 and 0.74), and T-cell-specific immune response (adjusted B: 0.89 and 0.99 ). Compared to PLWH with a time interval of $<21$ days between the first and second dose, those with an interval of 21-28 days and $>28$ days had higher neutralizing activity (adjusted B: 0.37 and 0.36), total antibody (adjusted B: 0.93 and 1.15), and S-IgG levels (adjusted B: 0.43 and 0.53) (Tables 6 and S2). A longer time since HIV diagnosis was correlated with higher CD4+ T-cell counts $(r=0.19, p=0.03)$. However, there was no significant correlation between HIV viral load and time since HIV diagnosis $(\mathrm{r}=-0.004$, $p=0.97$ ). 
Table 3. Seropositivity of SARS-CoV-2 neutralizing activity, total antibody, S-IgG, and T-cell-specific immune response among HIV-negative individuals and people living with HIV (PLWH) who had received at least one dose of SARS-CoV-2 vaccine.

\begin{tabular}{|c|c|c|c|c|c|c|c|c|c|c|c|c|}
\hline & \multicolumn{3}{|c|}{ Neutralizing Activity } & \multicolumn{3}{|c|}{ Total Antibody } & \multicolumn{3}{|c|}{ S-IgG } & \multicolumn{3}{|c|}{ T-Cell-Specific Immune Response } \\
\hline & PLWH & $\begin{array}{c}\text { HIV- } \\
\text { Negative }\end{array}$ & $p$ & PLWH & $\begin{array}{c}\text { HIV- } \\
\text { Negative }\end{array}$ & $p$ & PLWH & $\begin{array}{c}\text { HIV- } \\
\text { Negative }\end{array}$ & $p$ & PLWH & $\begin{array}{c}\text { HIV- } \\
\text { Negative }\end{array}$ & $p$ \\
\hline & $\%(\mathrm{n} / \mathrm{N})$ & $\%(n / N)$ & & $\%(n / N)$ & $\%(n / N)$ & & $\%(n / N)$ & $\%(n / N)$ & & $\%(n / N)$ & $\%(n / N)$ & \\
\hline $\begin{array}{c}\text { Partially } \\
\text { vaccinated }\end{array}$ & $\begin{array}{c}5.7 \\
(2 / 35)\end{array}$ & $\begin{array}{c}50.0 \\
(1 / 2)\end{array}$ & 0.03 & $\begin{array}{c}31.4 \\
(11 / 35)\end{array}$ & $\begin{array}{c}50.0 \\
(1 / 2)\end{array}$ & 0.55 & $\begin{array}{c}31.4 \\
(11 / 35)\end{array}$ & $\begin{array}{c}100 \\
(2 / 2)\end{array}$ & 0.12 & $\begin{array}{c}20.0 \\
(7 / 35)\end{array}$ & $\begin{array}{c}50.0 \\
(1 / 2)\end{array}$ & 0.32 \\
\hline $\begin{array}{l}0-14 \text { days since } \\
\text { fully vaccinated }\end{array}$ & $\begin{array}{c}33.3 \\
(5 / 15)\end{array}$ & $\begin{array}{c}75 \\
(6 / 8)\end{array}$ & 0.09 & $\begin{array}{c}46.7 \\
(7 / 15)\end{array}$ & $\begin{array}{c}100 \\
(8 / 8)\end{array}$ & 0.01 & $\begin{array}{c}66.7 \\
(10 / 15)\end{array}$ & $\begin{array}{c}100 \\
(8 / 8)\end{array}$ & 0.09 & $\begin{array}{c}33.3 \\
(5 / 15)\end{array}$ & $\begin{array}{c}100 \\
(8 / 8)\end{array}$ & 0.01 \\
\hline $\begin{array}{l}\text { 15-28 days since } \\
\text { fully vaccinated }\end{array}$ & $\begin{array}{c}81.6 \\
(31 / 38)\end{array}$ & $\begin{array}{c}90.9 \\
(10 / 11)\end{array}$ & 0.66 & $\begin{array}{c}94.7 \\
(36 / 38)\end{array}$ & $\begin{array}{c}100 \\
(11 / 11)\end{array}$ & 0.60 & $\begin{array}{c}100 \\
(38 / 38)\end{array}$ & $\begin{array}{c}100 \\
(11 / 11)\end{array}$ & N.A. & $\begin{array}{c}65.8 \\
(25 / 38)\end{array}$ & $\begin{array}{c}81.8 \\
(9 / 11)\end{array}$ & 0.27 \\
\hline $\begin{array}{l}29-56 \text { days since } \\
\text { fully vaccinated }\end{array}$ & $\begin{array}{c}84.6 \\
(22 / 26)\end{array}$ & $\begin{array}{c}85.7 \\
(18 / 21)\end{array}$ & 0.62 & $\begin{array}{c}88.5 \\
(23 / 26)\end{array}$ & $\begin{array}{c}95.2 \\
(20 / 21)\end{array}$ & 0.41 & $\begin{array}{c}96.2 \\
(25 / 26)\end{array}$ & $\begin{array}{c}100 \\
(21 / 21)\end{array}$ & 0.55 & $\begin{array}{c}53.8 \\
(14 / 26)\end{array}$ & $\begin{array}{c}81.0 \\
(17.21)\end{array}$ & 0.049 \\
\hline $\begin{array}{l}57-84 \text { days since } \\
\text { fully vaccinated }\end{array}$ & $\begin{array}{c}58.3 \\
(7 / 12)\end{array}$ & $\begin{array}{c}100 \\
(3 / 3)\end{array}$ & 0.26 & $\begin{array}{c}66.7 \\
(8 / 12)\end{array}$ & $\begin{array}{l}100 \\
(3 / 3)\end{array}$ & 0.36 & $\begin{array}{c}91.7 \\
(11 / 12)\end{array}$ & $\begin{array}{c}100 \\
(3 / 3)\end{array}$ & 0.80 & $\begin{array}{c}16.7 \\
(2 / 12)\end{array}$ & $\begin{array}{l}100 \\
(3 / 3)\end{array}$ & 0.02 \\
\hline $\begin{array}{l}>84 \text { days since } \\
\text { fully vaccinated }\end{array}$ & $\begin{array}{l}66.7 \\
(2 / 3)\end{array}$ & $\begin{array}{l}62.5 \\
(5 / 8)\end{array}$ & 0.72 & $\begin{array}{c}100 \\
(3 / 3)\end{array}$ & $\begin{array}{c}100 \\
(8 / 8)\end{array}$ & N.A. & $\begin{array}{c}100 \\
(3 / 3)\end{array}$ & $\begin{array}{c}100 \\
(8 / 8)\end{array}$ & N.A. & $\begin{array}{l}33.3 \\
(1 / 3)\end{array}$ & $\begin{array}{c}50.0 \\
(4 / 8)\end{array}$ & 0.62 \\
\hline $\begin{array}{l}\text { Among all } \\
\text { participants }\end{array}$ & $\begin{array}{c}53.5 \\
(69 / 129)\end{array}$ & $\begin{array}{c}81.1 \\
(43 / 53)\end{array}$ & $<0.001$ & $\begin{array}{c}68.2 \\
(88 / 129)\end{array}$ & $\begin{array}{c}96.2 \\
(51 / 53)\end{array}$ & $<0.001$ & $\begin{array}{c}76.0 \\
(98 / 129)\end{array}$ & $\begin{array}{c}100 \\
(53 / 53)\end{array}$ & $<0.001$ & $\begin{array}{c}41.9 \\
(54 / 129)\end{array}$ & $\begin{array}{c}79.2 \\
(42 / 53)\end{array}$ & $<0.001$ \\
\hline $\begin{array}{c}\text { Among } \\
\text { participants who } \\
\text { were fully } \\
\text { vaccinated }\end{array}$ & $\begin{array}{c}71.3 \\
(67 / 94)\end{array}$ & $\begin{array}{c}82.4 \\
(42 / 51)\end{array}$ & 0.14 & $\begin{array}{c}81.9 \\
(77 / 94)\end{array}$ & $\begin{array}{c}98.0 \\
(50 / 51)\end{array}$ & 0.005 & $\begin{array}{c}96.2 \\
(87 / 94)\end{array}$ & $\begin{array}{c}100 \\
(51 / 51)\end{array}$ & 0.04 & $\begin{array}{c}50.0 \\
(47 / 94)\end{array}$ & $\begin{array}{c}80.4 \\
(41 / 51)\end{array}$ & $<0.001$ \\
\hline
\end{tabular}


Table 4. Levels of SARS-CoV-2 neutralizing activity, total antibody, S-IgG, and T-cell-specific immune response among HIV-negative individuals and people living with HIV (PLWH) who had received at least one dose of SARS-CoV-2 vaccine.

\begin{tabular}{|c|c|c|c|c|c|c|c|c|c|c|c|c|}
\hline & \multicolumn{3}{|c|}{ Neutralizing Activity } & \multicolumn{3}{|c|}{ Total Antibody } & \multicolumn{3}{|c|}{ S-IgG } & \multicolumn{3}{|c|}{ T-Cell-Specific Immune Response } \\
\hline & PLWH & $\begin{array}{l}\text { HIV- } \\
\text { Negative }\end{array}$ & $p$ & PLWH & $\begin{array}{l}\text { HIV- } \\
\text { Negative }\end{array}$ & $p$ & PLWH & $\begin{array}{c}\text { HIV- } \\
\text { Negative }\end{array}$ & $p$ & PLWH & $\begin{array}{c}\text { HIV- } \\
\text { Negative }\end{array}$ & $p$ \\
\hline & $\begin{array}{c}\text { GMT } \\
(95 \% \mathrm{CI})\end{array}$ & $\begin{array}{c}\text { GMT } \\
(95 \% \mathrm{CI})\end{array}$ & & $\begin{array}{l}\text { Median } \\
\text { (IQR) }\end{array}$ & $\begin{array}{l}\text { Median } \\
\text { (IQR) }\end{array}$ & & $\begin{array}{l}\text { Median } \\
\text { (IQR) }\end{array}$ & $\begin{array}{l}\text { Median } \\
\text { (IQR) }\end{array}$ & & $\begin{array}{l}\text { Median } \\
\text { (IQR) }\end{array}$ & $\begin{array}{l}\text { Median } \\
\text { (IQR) }\end{array}$ & \\
\hline $\begin{array}{c}\text { Partially } \\
\text { vaccinated }\end{array}$ & $\begin{array}{c}4.6 \\
(4.0,9.8)\end{array}$ & $\begin{array}{c}5.6 \\
\text { (N.A.) }\end{array}$ & 0.43 & $\begin{array}{c}0.2 \\
(0.02,1.1)\end{array}$ & $\begin{array}{c}2.1 \\
\text { (N.A.) }\end{array}$ & 0.20 & $\begin{array}{c}0.6 \\
(0.3,1.5)\end{array}$ & $\begin{array}{l}3.99 \\
\text { (N.A.) }\end{array}$ & 0.03 & $\begin{array}{c}6.4 \\
(0.2,26.7)\end{array}$ & $\begin{array}{l}36.2 \\
\text { (N.A.) }\end{array}$ & 0.16 \\
\hline $\begin{array}{l}0-14 \text { days since } \\
\text { fully vaccinated }\end{array}$ & $\begin{array}{c}8.5 \\
(4.0,64.6)\end{array}$ & $\begin{array}{c}31.6 \\
(4.0,257.0)\end{array}$ & 0.03 & $\begin{array}{c}0.8 \\
(0.03,16.8)\end{array}$ & $\begin{array}{c}104.8 \\
(7.4,279.5)\end{array}$ & 0.01 & $\begin{array}{c}3.1 \\
(1.1,16.2)\end{array}$ & $\begin{array}{c}11.9 \\
(5.1,55.5)\end{array}$ & 0.04 & $\begin{array}{c}5.3 \\
(0.1,88.8)\end{array}$ & $\begin{array}{c}413.6 \\
(91.8,575.5)\end{array}$ & 0.001 \\
\hline $\begin{array}{l}\text { 15-28 days since } \\
\text { fully vaccinated }\end{array}$ & $\begin{array}{c}24.0 \\
(4.0,380.2)\end{array}$ & $\begin{array}{c}23.4 \\
(4.0,64.0)\end{array}$ & 0.97 & $\begin{array}{c}28.9 \\
(7.4,83.2)\end{array}$ & $\begin{array}{c}40.3 \\
(28.5,71.6)\end{array}$ & 0.24 & $\begin{array}{c}9.0 \\
(4.6,16.0)\end{array}$ & $\begin{array}{c}13.9 \\
(10.1,32.0)\end{array}$ & 0.13 & $\begin{array}{c}56.08 \\
(19.6,118.7)\end{array}$ & $\begin{array}{c}91.54 \\
(31.1,227.4)\end{array}$ & 0.29 \\
\hline $\begin{array}{l}29-56 \text { days since } \\
\text { fully vaccinated }\end{array}$ & $\begin{array}{c}14.1 \\
(4.0,64.6)\end{array}$ & $\begin{array}{c}20.9 \\
(4.0,190.5)\end{array}$ & 0.24 & $\begin{array}{c}11.8 \\
(5.7,27.3)\end{array}$ & $\begin{array}{c}42.7 \\
(8.4,74.9)\end{array}$ & 0.04 & $\begin{array}{c}7.2 \\
(4.5,12.2)\end{array}$ & $\begin{array}{c}9.6 \\
(7.2,21.9)\end{array}$ & 0.03 & $\begin{array}{c}37.2 \\
(6.4,121.1)\end{array}$ & $\begin{array}{c}63.6 \\
(35.4,182.1)\end{array}$ & 0.13 \\
\hline $\begin{array}{l}57-84 \text { days since } \\
\text { fully vaccinated }\end{array}$ & $\begin{array}{c}11.0 \\
(4.0,95.5)\end{array}$ & $\begin{array}{c}26.3 \\
(12.0,64.0)\end{array}$ & 0.18 & $\begin{array}{c}6.2 \\
(0.5,11.7)\end{array}$ & $\begin{array}{c}33.4 \\
\text { (N.A.) }\end{array}$ & 0.04 & $\begin{array}{c}3.4 \\
(1.4,5.7)\end{array}$ & $\begin{array}{l}10.5 \\
\text { (N.A.) }\end{array}$ & 0.03 & $\begin{array}{c}3.6 \\
(0.1,17.1)\end{array}$ & $\begin{array}{l}205.5 \\
\text { (N.A.) }\end{array}$ & 0.08 \\
\hline $\begin{array}{l}\text { Among all } \\
\text { participants }\end{array}$ & $\begin{array}{c}11.0 \\
(4.0,95.5)\end{array}$ & $\begin{array}{c}20.0 \\
(4.0,190.5)\end{array}$ & 0.001 & $\begin{array}{c}5.6 \\
(0.4,25.2)\end{array}$ & $\begin{array}{c}32.6 \\
(8.4,72.3)\end{array}$ & $<0.001$ & $\begin{array}{c}4.3 \\
(1.2,10.0)\end{array}$ & $\begin{array}{c}9.6 \\
(5.4,18.9)\end{array}$ & $<0.001$ & $\begin{array}{c}18.7 \\
(2.4,77.9)\end{array}$ & $\begin{array}{c}63.6 \\
(36.0,226.4)\end{array}$ & $<0.001$ \\
\hline $\begin{array}{c}\text { Among } \\
\text { participants who } \\
\text { were fully } \\
\text { vaccinated }\end{array}$ & $\begin{array}{c}15.1 \\
(4.0,128.8)\end{array}$ & $\begin{array}{c}20.9 \\
(4.0,190.5)\end{array}$ & 0.09 & $\begin{array}{c}10.3 \\
(2.3,38.8)\end{array}$ & $\begin{array}{c}33.4 \\
(10.1,73.0)\end{array}$ & $<0.001$ & $\begin{array}{c}6.8 \\
(3.3,12.1)\end{array}$ & $\begin{array}{c}10.1 \\
(6.5,19.4)\end{array}$ & 0.007 & $\begin{array}{c}30.6 \\
(5.2,103.2)\end{array}$ & $\begin{array}{c}68.4 \\
(36.1,227.4)\end{array}$ & 0.001 \\
\hline
\end{tabular}


Table 5. Comparing immunogenicity indicator levels between different subgroups of people living with HIV (PLWH) and HIV-negative individuals.

\begin{tabular}{|c|c|c|c|c|c|c|c|c|}
\hline & \multicolumn{2}{|c|}{ Neutralizing Activity } & \multicolumn{2}{|c|}{ Total Antibody } & \multicolumn{2}{|l|}{ S-IgG } & \multicolumn{2}{|c|}{ T-Cell-Specific Immune Response } \\
\hline & $\begin{array}{l}\text { Adjusted B } \\
(95 \% \mathrm{CI})\end{array}$ & $p$ Values & $\begin{array}{l}\text { Adjusted B } \\
(95 \% \mathrm{CI})\end{array}$ & $p$ Values & $\begin{array}{l}\text { Adjusted B } \\
(95 \% \mathrm{CI})\end{array}$ & $p$ Values & $\begin{array}{c}\text { Adjusted B } \\
(95 \% \mathrm{CI})\end{array}$ & $p$ Values \\
\hline $\begin{array}{l}\text { Reference 1: HIV-negative } \\
\text { individuals }(n=53)\end{array}$ & Ref & Ref & Ref & Ref & Ref & Ref & Ref & Ref \\
\hline PLWH $(n=129)$ & $-0.18(-0.36,-0.001)$ & 0.049 & $-0.80(-1.15,-0.46)$ & $<0.001$ & $-0.31(-0.51,-0.12)$ & 0.002 & $-0.64(-1.05,-0.23)$ & 0.002 \\
\hline $\begin{array}{c}\text { PLWH with CD4 }{ }^{+} \text {T-cell } \\
\text { counts }<500 \text { and } \\
\text { detectable viral load } \\
(n=13)\end{array}$ & $-0.69(-1.02,-0.36)$ & $<0.001$ & $-2.51(-3.01,-2.01)$ & $<0.001$ & $-1.16(-1.44,-0.88)$ & $<0.001$ & $-1.35(-1.94,-0.76)$ & $<0.001$ \\
\hline $\begin{array}{l}\text { PLWH with CD4 }{ }^{+} \text {T-cell } \\
\text { counts }<500 \text { and } \\
\text { undetectable viral load } \\
(n=19)\end{array}$ & $-0.21(-0.40,-0.01)$ & 0.04 & $-1.05(-1.57,-0.54)$ & $<0.001$ & $-0.38(-0.67,-0.09)$ & 0.01 & $-0.91(-1.39,-0.42)$ & $<0.001$ \\
\hline $\begin{array}{l}\text { PLWH with CD4+ T-cell } \\
\text { counts } \geq 500 \text { and } \\
\text { detectable viral load } \\
(n=41)\end{array}$ & $-0.32(-0.57,-0.08)$ & 0.01 & $-1.32(-1.81,-0.82)$ & $<0.001$ & $-0.72(-1.02,-0.42)$ & $<0.001$ & $-0.98(-1.41,-0.55)$ & $<0.001$ \\
\hline $\begin{array}{l}\text { PLWH with CD4+ T-cell } \\
\text { counts } \geq 500 \text { and } \\
\text { undetectable viral load } \\
(n=56)\end{array}$ & $-0.23(-0.57,0.10)$ & 0.16 & $-0.72(-1.05,-0.40)$ & $<0.001$ & $-0.32(-0.51,-0.13)$ & 0.001 & $-0.46(-0.84,-0.08)$ & 0.02 \\
\hline $\begin{array}{c}\text { Reference } 2 \text { : Fully } \\
\text { vaccinated HIV-negative } \\
\text { individuals }(n=51)\end{array}$ & Ref & Ref & Ref & Ref & Ref & Ref & Ref & Ref \\
\hline $\begin{array}{l}\text { Fully vaccinated PLWH } \\
\qquad(n=94)\end{array}$ & $-0.15(-0.35,0.04)$ & 0.13 & $-0.68(-1.03,-0.33)$ & $<0.001$ & $-0.27(-0.48,-0.07)$ & 0.01 & $-0.61(-1.00,-0.22)$ & 0.002 \\
\hline
\end{tabular}

Ref: reference group. Adjusted B: adjusted correlation coefficients, adjusted for background characteristics with significant between-group differences in Table 1 (age group, gender presence of chronic conditions other than HIV, types of SARS-CoV-2 vaccine, time interval between first and second dose, and SARS-CoV-2 vaccination status). 
Table 6. Factors associated with SARS-CoV-2 total antibody, neutralizing antibody, S-IgG, and T-cell-specific immune response levels among people living with HIV (PLWH) $(n=129)$.

\begin{tabular}{|c|c|c|c|c|c|c|c|c|}
\hline & \multicolumn{2}{|c|}{ Total Antibody } & \multicolumn{2}{|c|}{ Neutralizing Activity } & \multicolumn{2}{|c|}{ S-IgG } & \multicolumn{2}{|c|}{$\begin{array}{l}\text { T-Cell-Specific Immune } \\
\text { Response }\end{array}$} \\
\hline & $\begin{array}{c}\text { Adjusted B } \\
(95 \% C I)\end{array}$ & $p$ Values & $\begin{array}{c}\text { Adjusted B } \\
(95 \% \mathrm{CI})\end{array}$ & $p$ Values & $\begin{array}{c}\text { Adjusted B } \\
(95 \% C I)\end{array}$ & $p$ Values & $\begin{array}{c}\text { Adjusted B } \\
(95 \% \mathrm{CI})\end{array}$ & $p$ Values \\
\hline \multicolumn{9}{|l|}{ Characteristics related to HIV infection } \\
\hline \multicolumn{9}{|l|}{ Years since HIV diagnosis (years) } \\
\hline$\leq 1$ & Ref & Ref & Ref & Ref & & & & \\
\hline $2-5$ & $\begin{array}{c}0.71 \\
(0.23,1.19)\end{array}$ & 0.004 & $\begin{array}{c}0.27 \\
(0.05,0.48)\end{array}$ & 0.02 & & & & \\
\hline $6-10$ & $\begin{array}{c}0.49 \\
(-0.03,1.00)\end{array}$ & 0.07 & $\begin{array}{c}0.23 \\
(-0.01,0.46)\end{array}$ & 0.06 & & & & \\
\hline$>10$ & $\begin{array}{c}0.44 \\
(-0.13,1.05)\end{array}$ & 0.13 & $\begin{array}{c}0.15 \\
(-0.11,0.20)\end{array}$ & 0.24 & - & - & - & - \\
\hline \multicolumn{9}{|l|}{ Viral load $(\mathrm{cp} / \mathrm{mL})$} \\
\hline Undetectable & Ref & Ref & & & Ref & Ref & Ref & Ref \\
\hline 61-200 & $\begin{array}{c}-0.24 \\
(-0.61,0.14)\end{array}$ & 0.21 & & & $\begin{array}{c}-0.19 \\
(-0.40,0.03)\end{array}$ & 0.08 & $\begin{array}{c}-0.01 \\
(-0.44,0.42)\end{array}$ & 0.97 \\
\hline$>200$ & $\begin{array}{c}-0.24 \\
(-0.69,0.22)\end{array}$ & 0.31 & - & & $\begin{array}{c}-0.24 \\
(-0.50,0.03)\end{array}$ & 0.08 & $\begin{array}{c}-0.27 \\
(-0.78,0.25)\end{array}$ & 0.30 \\
\hline \multicolumn{9}{|l|}{ CD4+ T-cell count (cells $/ \mu \mathrm{L})$} \\
\hline$<500$ & & & & & & & Ref & Ref \\
\hline $500-1000$ & & & & & & & $\begin{array}{c}0.47 \\
(0.05,0.89)\end{array}$ & 0.03 \\
\hline$>1000$ & - & - & - & - & - & - & $\begin{array}{c}0.40 \\
(-0.22,1.01)\end{array}$ & 0.20 \\
\hline \multicolumn{9}{|l|}{ On ART } \\
\hline Yes & - & - & - & - & - & - & - & - \\
\hline \multicolumn{9}{|l|}{ SARS-CoV-2 vaccination } \\
\hline Partially vaccinated & Ref & Ref & Ref & Ref & Ref & Ref & Ref & Ref \\
\hline 0-14 days since fully vaccinated & $0.03(-0.36,0.63)$ & 0.92 & $\begin{array}{c}0.06 \\
(-0.21,0.32)\end{array}$ & 0.68 & $\begin{array}{c}0.21 \\
(-0.13,0.54)\end{array}$ & 0.022 & $\begin{array}{c}0.16 \\
(-0.46,0.78)\end{array}$ & 0.60 \\
\hline 15-28 days since fully vaccinated & $\begin{array}{c}1.03 \\
(0.43,1.57)\end{array}$ & 0.001 & $\begin{array}{c}0.35 \\
(0.08,0.63)\end{array}$ & 0.01 & $\begin{array}{c}0.74 \\
(0.39,1.08)\end{array}$ & $<0.001$ & $\begin{array}{c}0.99 \\
(0.50,1.47)\end{array}$ & $<0.001$ \\
\hline
\end{tabular}


Table 6. Cont.

\begin{tabular}{|c|c|c|c|c|c|c|c|c|}
\hline & \multicolumn{2}{|c|}{ Total Antibody } & \multicolumn{2}{|c|}{ Neutralizing Activity } & \multicolumn{2}{|c|}{ S-IgG } & \multicolumn{2}{|c|}{$\begin{array}{l}\text { T-Cell-Specific Immune } \\
\text { Response }\end{array}$} \\
\hline & $\begin{array}{l}\text { Adjusted B } \\
(95 \% \mathrm{CI})\end{array}$ & $p$ Values & $\begin{array}{l}\text { Adjusted B } \\
(95 \% \mathrm{CI})\end{array}$ & $p$ Values & $\begin{array}{l}\text { Adjusted B } \\
(95 \% \mathrm{CI})\end{array}$ & $p$ Values & $\begin{array}{l}\text { Adjusted B } \\
(95 \% \mathrm{CI})\end{array}$ & $p$ Values \\
\hline 29-56 days since fully vaccinated & $\begin{array}{c}1.03 \\
(0.47,1.59)\end{array}$ & $<0.001$ & $\begin{array}{c}0.21 \\
(-0.09,0.46)\end{array}$ & 0.10 & $\begin{array}{c}0.73 \\
(0.41,1.05)\end{array}$ & $<0.001$ & $\begin{array}{c}0.89 \\
(0.37,1.40)\end{array}$ & 0.001 \\
\hline 57-84 days since fully vaccinated & $\begin{array}{c}0.85 \\
(0.15,1.55)\end{array}$ & 0.01 & $\begin{array}{c}0.19 \\
(-0.11,0.49)\end{array}$ & 0.21 & $\begin{array}{c}0.47 \\
(0.09,0.84)\end{array}$ & 0.02 & $\begin{array}{c}-0.10 \\
(-0.76,0.57)\end{array}$ & 0.77 \\
\hline$>84$ days since fully vaccinated & $\begin{array}{c}0.29 \\
(-0.83,1.41)\end{array}$ & 0.58 & $\begin{array}{c}-0.25 \\
(-0.77,0.26)\end{array}$ & 0.34 & $\begin{array}{c}0.27 \\
(-0.37,0.93)\end{array}$ & 0.41 & $\begin{array}{c}0.20 \\
(-1.01,1.41)\end{array}$ & 0.74 \\
\hline $\begin{array}{c}\text { Type of SARS-CoV-2 vaccine } \\
\text { Sinopharm }\end{array}$ & Ref & Ref & Ref & Ref & Ref & Ref & & \\
\hline Sinovac CoronaVac & $\begin{array}{c}0.26 \\
(-0.07,0.59)\end{array}$ & 0.12 & $\begin{array}{c}0.05 \\
(-0.10,0.20)\end{array}$ & 0.50 & $\begin{array}{c}0.07 \\
(-0.11,0.25)\end{array}$ & 0.45 & - & - \\
\hline \multicolumn{9}{|l|}{$\begin{array}{l}\text { Time interval (days) between the first and } \\
\text { second dose }\end{array}$} \\
\hline$<21$ days & Ref & Ref & Ref & Ref & Ref & Ref & & \\
\hline $21-28$ days & $\begin{array}{c}0.93 \\
(0.43,1.43)\end{array}$ & $<0.001$ & $\begin{array}{c}0.37 \\
(0.15,0.59)\end{array}$ & 0.001 & $\begin{array}{c}0.43 \\
(0.14,0.71)\end{array}$ & 0.003 & & \\
\hline Not applicable (partially vaccinated) & N.A. & N.A. & N.A. & N.A. & N.A. & N.A. & - & - \\
\hline
\end{tabular}

Ref: reference group. Adjusted B: adjusted coefficients obtained from multivariate linear regression models using all significant variables as candidates. - indicates $p>0.05$ in univariate analysis and exclusion from multivariate analysis. 


\section{Discussion}

Understanding the differences in immunoresponse between HIV-negative and HIVpositive individuals is essential in planning SARS-CoV-2 vaccination for PLWH. We found the levels of adverse events to be comparable between PLWH and HIV-negative individuals. Although there was no significant between-group difference in seropositivity or levels of neutralizing activity, fully vaccinated PLWH had lower seropositivity and lower levels of total antibody, S-IgG, and T-cell-specific immune responses than fully vaccinated HIV-negative individuals. Our findings fill the knowledge gap concerning immune responses to SARS-CoV-2 vaccines among PLWH. They contribute critical evidence for policymaking and vaccination-program planning for countries that mainly use inactivated SARS-CoV-2 vaccines.

Similar to studies on mRNA/adenovirus vector SARS-CoV-2 vaccines [20-24], there was no between-group difference in prevalence $(p=0.19)$ or severity $(p=0.13-0.77)$ of self-reported adverse events. Most of the reported adverse events were very mild $/ \mathrm{mild}$ among PLWH (41-100\%). Therefore, inactivated SARS-CoV-2 vaccines are safe for PLWH.

Four immunogenicity indicator levels were significantly lower among PLWH at 0-14 days after receiving the second dose. PLWH might take longer to develop humoral and cellular immune responses to inactivated SARS-CoV-2 vaccines. Previous case reports observed a prolonged course of antibody development among PLWH infected with SARSCoV-2 [32]. The studied immunogenicity indicators peaked at 15-56 days after the second dose among PLWH, a finding in line with previous research conducted with HIV-negative individuals and PLWH who received other SARS-CoV-2 vaccines [20-24]. However, the peak levels of these indicators were lower among PLWH, especially for total antibody and S-IgG. A faster decline in immune responses was also observed among PLWH. All four immunogenicity indicator levels declined $>56$ days after receiving the second dose among PLWH, while these indicators remained stable among HIV-negative individuals even 84 days after the second dose. This study observed significantly lower total antibody and S-IgG levels among PLWH $>56$ days after the second dose. B-cell dysfunction caused by HIV gp120 binding directly to primary B cells, and impaired cellular immunity caused by CD4+ T-cell depletion among PLWH, might explain the slower development, lower peak levels, and faster decline of both humoral and cellular immune responses to SARS-CoV-2 vaccines that we observed $[33,34]$. Such findings indicate that PLWH might need a booster dose after the primary series and might need it earlier than HIV-negative individuals. Future studies with large sample sizes are needed to investigate long-term changes in these immunogenicity indicators among PLWH.

Neutralizing antibody plays an important role in SARS-CoV-2 clearance and is a key indicator of protection after vaccination [35]. We found that seropositivity and levels of neutralizing activity were similar among fully vaccinated PLWH and HIV-negative individuals. This implies that both groups obtained good protection against SARS-CoV-2 after the vaccination and PLWH should complete both doses of vaccination as required. Subgroup analysis showed that PLWH with higher CD4+ T-cell counts and undetectable viral loads did not have significantly lower neutralizing activity levels than HIV-negative individuals, a finding in line with previous studies examining mRNA and/or adenovirus vector SARS-CoV-2 vaccines [20-24]. However, PLWH with lower CD4+ T-cell counts $(<500)$ and/or detectable viral loads had lower neutralizing activity levels. Such findings add to knowledge regarding immune responses to SARS-CoV-2 vaccines among PLWH with more severe immunodeficiency. PLWH with more severe immunodeficiency should be encouraged to receive SARS-CoV-2 vaccines. In contrast to findings regarding other types of vaccines, our study observed significantly lower total antibody, S-IgG, and T-cell-specific immune response levels among PLWH compared to HIV-negative individuals. These differences could not be fully explained as a result of the larger proportion of PLWH with low CD4+ T-cell counts or detectable HIV viral loads in this study. These indicators were lower among PLWH regardless of their CD4+ T-cell counts or HIV viral loads. Future 
studies should compare PLWH's immunogenicity in response to different types of SARS$\mathrm{CoV}-2$ vaccines in order to determine the optimal choice of vaccine for PLWH.

Compared to newly diagnosed PLWH, those who had been diagnosed 2-5 years prior to the commencement of the study had higher neutralizing activity and total antibody levels. Since increased length of time since HIV diagnosis was correlated with higher CD4+ T-cell counts, it is possible that these PLWH had better-functioning immune systems and hence higher neutralizing activity and antibody titers. This finding also highlights the need to further increase HIV testing coverage among key populations to improve early identification of HIV infection and direct these individuals toward treatment and care, hence improving the effectiveness of SARS-CoV-2 vaccination for PLWH. Moreover, our results also suggest that PLWH who had a longer interval between the first and second dose (21-28 days or $>28$ days) had significantly higher neutralizing activity, total antibody, and S-IgG levels compared to those with a shorter interval between doses. Existing guidelines regarding SARS-CoV-2 vaccination for PLWH do not mention the optimal vaccination interval. Our findings suggest that future SARS-CoV-2 vaccination programs for PLWH should consider a longer interval between doses. However, more research is needed to confirm the optimal interval between doses for PLWH.

This study has several strengths. First, we measured neutralizing activity using the gold-standard neutralization assay. Second, all participants underwent humoral and cellular immune response analyses in this study. Third, this study included a diverse sample of PLWH with different CD4+ T-cell levels and HIV viral loads, helping to fill the knowledge gap regarding the immunogenicity of SARS-CoV-2 vaccines among PLWH with impaired immune system functioning and poorer control of HIV. Fourth, the impact of between-group differences in background characteristics on immunogenicity may have been reduced in this study, since background characteristics were controlled during the comparison. Furthermore, this is also one the first studies to assess relationships between characteristics of PLWH and their immunogenicity in response to SARS-CoV-2 vaccines.

This study also has some limitations. Gender imbalance was one such factor. Gender is a biological variable that affects the functioning of the immune system. Generally, adult females mount stronger innate and adaptive immune responses than males, as noted in [36]; however, this previous study did not show any difference in immunogenicity in response to SARS-CoV-2 vaccination between males and females. Second, this was a cross-sectional study. Possible changes in immunogenicity indicator levels over time were unclear. Such a study design is also unable to establish causal relationships. Third, we did not match the sample of HIV-negative individuals according to the PLWH's characteristics. There are significant between-group differences in socio-demographics, presence of other chronic conditions, and vaccination characteristics. However, we controlled these characteristics when comparing the between-group differences in immunogenicity. In addition, the presence and severity of adverse events were self-reported by participants and might have been subject to recall bias. This means we were also unable to compare the safety data with other studies that used clinician assessments.

\section{Conclusions}

Inactivated SARS-CoV-2 vaccines are safe for PLWH. Although there was no difference in the level of neutralizing activity, fully vaccinated PLWH had significantly lower total antibody, S-IgG, and T-cell-specific immune response levels than fully vaccinated HIVnegative individuals. The immunogenicity indicator levels peaked $15-56$ days after PLWH receiving the second dose. A longer time since diagnosis and a longer interval between the first and second dose were correlated with better immune responses among PLWH. Future studies should compare PLWH's immunogenicity in response to different types of vaccines, assess immune responses over a longer term, and investigate the optimal interval between doses for this population. 
Supplementary Materials: The following are available online at https:/ / www.mdpi.com/article/ 10.3390/v14020277/s1. Table S1: Comparing seropositivity of immunogenicity indicators between different subgroups of PLWHA and HIV-negative individuals. Table S2: Unadjusted correlation coefficients of factors associated with SARS-CoV-2 total antibody, neutralizing activity, S-IgG, and T-cell-specific immune response levels among people living with HIV (PLWH) ( $n=129)$.

Author Contributions: Conceptualization: W.T., L.W., T.Z., Z.W., and J.X.; Methodology: W.T., L.W., T.Z., Z.W., and J.X.; Data curation: X.H., Y.Y., B.S., and D.X.; Formal analysis: Z.W. and Y.F.; Project administration: X.H., Y.Y., B.S., D.X., M.Y., X.J., J.D., X.Z., and S.Z.; Resources: X.H., Y.Y., B.S., D.X., M.Y., X.J., J.D., X.Z., and S.Z.; Supervision: J.X.; Writing-original draft preparation: Z.W., Y.F., and J.X.; Writing-review and editing: Z.W., Y.F., and J.X.; Funding acquisition: X.H., B.S., L.W. and J.X. All authors have read and agreed to the published version of the manuscript.

Funding: This study was supported by the Beijing Excellent Talent Plan (2018000021223ZK04 to X.H.), the Beijing Talent Project in the New Millennium (2020A35 to X.H.), the National Natural Science Foundation of China (81903371 to W.T.; 81772165 and 81974303 to B.S.), the National 13th Five-Year Grand Program on Key Infectious Disease Control (2017ZX10202102-005-003 to B.S.), the China Primary Health Care Foundation-Youan Medical Development Fund (BJYAYY-2020PY-01 to B.S.), the Beijing Key Laboratory for HIV / AIDS Research (BZ0089 to B.S.) and The Beijing Natural Science Foundation-Haidian Original Innovation Joint Fund (L212012 to L.W.). Funders had no role in the design, data collection, analysis, or interpretation of the study, or in the preparation of the manuscript.

Institutional Review Board Statement: Ethics approval was obtained from the Institutional Review Boards of Changzhi Medical College (No. RT2021002, approval date: 2021-2-2) and Beijing Youan Hospital Research Ethics Committee (No. 2021-031, approval date: 2021-4-21)

Informed Consent Statement: Written informed consent was obtained from all participants before their study participation in accordance with the Declaration of Helsinki.

Data Availability Statement: The individual participant data used in this analysis are available upon request. Requests should be directed to the corresponding author and requesters must sign a data access and confidentiality agreement.

Acknowledgments: The authors would like to express their gratitude to all the participants for their engagement in this study.

Conflicts of Interest: The authors declare no conflict of interest.

\section{References}

1. UNAIDS. UNAIDS Data 2020. Available online: https://aids2020.unaids.org/report/ (accessed on 23 April 2021).

2. Cohen, M.S.; Chen, Y.Q.; McCauley, M.; Gamble, T.; Hosseinipour, M.C.; Kumarasamy, N.; Hakim, J.G.; Kumwenda, J.; Grinsztejn, B.; Pilotto, J.H.; et al. Prevention of HIV-1 Infection with Early Antiretroviral Therapy. N. Engl. J. Med. 2011, 365, 493-505. [CrossRef]

3. Plettenberg, A.; Brockmeyer, N.H.; Haastert, B.; Michalik, C.; Dupke, S.; Schewe, K.; Rausch, M.; Hower, M.; Ulmer, A.; Wolf, E.; et al. Impact of earlier HAART initiation on the immune status and clinical course of treated patients on the basis of cohort data of the German Competence Network for HIV/AIDS. Infection 2011, 39, 3-12. [CrossRef] [PubMed]

4. Yang, X.; Su, B.; Zhang, X.; Liu, Y.; Wu, H.; Zhang, T. Incomplete immune reconstitution in HIV/AIDS patients on antiretroviral therapy: Challenges of immunological non-responders. J. Leukoc. Biol. 2020, 107, 597-612. [CrossRef] [PubMed]

5. World Health Organization. Coronavirus Disease-Answers. Available online: https://www.who.int/emergencies/ diseases / novel-coronavirus-2019/ coronavirus-disease-answers?query=hiv\&referrerPageUrl=https \%3A\%2F\%2Fwww.who. int\%2Femergencies\%2Fdiseases\%2Fnovel-coronavirus-2019\%2Fcoronavirus-disease-answers (accessed on 27 December 2021).

6. Vanetti, C.; Trabattoni, D.; Stracuzzi, M.; Amendola, A.; Fappani, C.; Rubinacci, V.; Gianalio, L.; Biasin, M.; Dighera, A.; Saulle, I.; et al. Immunological characterization of HIV and SARS-CoV-2 coinfected young individuals. Cells 2021, 10, 3187. [CrossRef] [PubMed]

7. Mirzaei, H.; McFarland, W.; Karamouzian, M.; Sharifi, H. COVID-19 among People Living with HIV: A Systematic Review. AIDS Behav. 2021, 25, 1-8. [CrossRef]

8. Costenaro, P.; Minotti, C.; Barbieri, E.; Giaquinto, C.; Dona, D. SARS-CoV-2 infection in people living with HIV: A systematic review. Rev. Med. Virol. 2021, 31, 1-12. [CrossRef] [PubMed]

9. Cooper, T.J.; Woodward, B.L.; Alom, S.; Harky, A. Coronavirus disease 2019 (COVID-19) outcomes in HIV / AIDS patients. HIV Med. 2020, 21, 567-577. [CrossRef] 
10. Zheng, Z.; Peng, F.; Xu, B.; Zhao, J.; Liu, H.; Peng, J.; Li, Q.; Jiang, C.; Zhou, Y.; Liu, S.; et al. Risk factors of critical \& mortal COVID-19 cases: A systematic literature review and meta-analysis. J. Infect. 2020, 81, e16-e25. [CrossRef] [PubMed]

11. Prabhu, S.; Poongulali, S.; Kumarasamy, N. Impact of COVID-19 on people living with HIV: A review. J. Virus Erad. 2020, 6, 100019. [CrossRef] [PubMed]

12. Murdaca, G.; Noberasco, G.; Olobardi, D.; Lunardi, C.; Maule, M.; Delfina, L.; Triggiani, M.; Cardamone, C.; Befaremo, D.; Moroncini, G.; et al. Current take on systematic sclerosis patients' vaccination recommendations. Vaccines 2021, 9, 1426. [CrossRef]

13. National Health Commission of the People's Republic of China. Technical Guideline on COVID-19 Vaccination (1st Edition). Available online: http:/ / www.nhc.gov.cn/xcs/yqfkdt/202103/c2febfd04fc5498f916b1be080905771.shtml (accessed on 30 March 2021).

14. World Health Organization. Coronavirus Disease (COVID-19): Vaccines. Available online: https://www.who.int/news-room/ q-a-detail/coronavirus-disease-(covid-19)-vaccines?adgroupsurvey=\{adgroupsurvey $\}$ ggclid=EAIaIQobChMItc-btpTY8 gIVFBLnCh2CHw5rEAAYASAAEgIeBfD_BwE (accessed on 30 August 2021).

15. United States Department of Health and Human Services. Guidance for COVID-19 and People with HIV. Available online: https: // clinicalinfo.hiv.gov/sites/default/files/guidelines/documents/HIV_COVID_19_GL_2021.pdf (accessed on 19 August 2021).

16. Polack, F.P.; Thomas, S.J.; Kitchin, N.; Absalon, J.; Gurtman, A.; Lockhart, S.; Perez, J.L.; Pérez Marc, G.; Moreira, E.D.; Zerbini, C.; et al. Safety and efficacy of the BNT162b2 mRNA COVID-19 vaccine. N. Engl. J. Med. 2020, 383, 2603-2615. [CrossRef] [PubMed]

17. Voysey, M.; Clemens, S.A.C.; Madhi, S.A.; Weckx, L.Y.; Folegatti, P.M.; Aley, P.K.; Angus, B.; Baillie, V.L.; Barnabas, S.L.; Bhorat, Q.E.; et al. Safety and efficacy of the ChAdOx1 $\mathrm{nCoV}-19$ vaccine (AZD1222) against SARS-CoV-2: An interim analysis of four randomised controlled trials in Brazil, South Africa, and the UK. Lancet 2021, 397, 99-111. [CrossRef]

18. Baden, L.R.; El Sahly, H.M.; Essink, B.; Kotloff, K.; Frey, S.; Novak, R.; Diemert, D.; Spector, S.A.; Rouphael, N.; Creech, C.B.; et al. Efficacy and Safety of the mRNA-1273 SARS-CoV-2 Vaccine. N. Engl. J. Med. 2021, 384, 403-416. [CrossRef]

19. Sadoff, J.; Gray, G.; Vandebosch, A.; Cárdenas, V.; Shukarev, G.; Grinsztejn, B.; Goepfert, P.A.; Truyers, C.; Fennema, H.; Spiessens, B.; et al. Safety and Efficacy of Single-Dose Ad26.COV2.S Vaccine against Covid-19. N. Engl. J. Med. 2021, 384, $2187-2201$. [CrossRef] [PubMed]

20. Hosein, S.R. Encouraging Results from the Pfizer-BioNTech COVID-19 Vaccine in HIV-positive People. Available online: https: //www.catie.ca/en/catienews/2021-05-25/encouraging-results-pfizer-biontech-covid-19-vaccine-hiv-positive-people (accessed on 11 August 2021).

21. Ruddy, J.A.; Boyarsky, B.J.; Bailey, J.R.; Karaba, A.H.; Garonzik-Wang, J.M.; Segev, D.L.; Durand, C.M.; Werbel, W.A. Safety and antibody response to two-dose SARS-CoV-2 messenger RNA vaccination in persons with HIV. Aids 2021, 15, $2399-2401$. [CrossRef]

22. Woldemeskel, B.A.; Karaba, A.H.; Garliss, C.C.; Beck, E.J.; Wang, K.H.; Laeyendecker, O.; Cox, A.L.; Blankson, J.N. The BNT162b2 mRNA Vaccine Elicits Robust Humoral and Cellular Immune Responses in People Living with HIV. Clin. Infect. Dis. 2021, ciab648. [CrossRef]

23. Frater, J.; Ewer, K.J.; Ogbe, A.; Pace, M.; Adele, S.; Adland, E.; Alagaratnam, J.; Aley, P.K.; Ali, M.; Ansari, M.A.; et al. Safety and immunogenicity of the ChAdOx1 nCoV-19 (AZD1222) vaccine against SARS-CoV-2 in HIV infection: A single-arm substudy of a phase 2/3 clinical trial. Lancet HIV 2021, 8, e474-e485. [CrossRef]

24. Madhi, S.; Koen, A.; Fairlie, L.; Cutland, C.; Ballie, V.; Padayachee, S.; Dheda, K.; Barnabas, S.; Bhorat, Q.E.; Briner, C.; et al. ChAdOx1 nCoV-19 (AZD1222) Vaccine in People Living with and without HIV. Research Square. Available online: https: / / www.researchsquare.com/article/rs-322470/v1 (accessed on 11 August 2021).

25. World Health Organization. The Sinopharm COVID-19 Vaccine: What You Need to Know. Available online: https://www.who. int/news-room/feature-stories/detail/the-sinopharm-covid-19-vaccine-what-you-need-to-know (accessed on 27 August 2021).

26. World Health Organization. The Sinovac COVID-19 Vaccine: What You Need to Know. Available online: https://www.who.int/ news-room/feature-stories/detail/the-sinovac-covid-19-vaccine-what-you-need-to-know (accessed on 27 August 2021).

27. The state Council the People's Republic of China. Over Three Billion Doses of SARS-CoV-2 Vaccines Were Manufactured by China in 2021. Available online: http:/ / www.gov.cn/xinwen/2021-04/21/content_5600940.htm (accessed on 30 August 2021).

28. Huang, X.; Yu, M.; Fu, G.; Lan, G.; Li, L.; Yang, J.; Qiao, Y.; Zhao, J.; Qian, H.-Z.; Zhang, X.; et al. Willingness to Receive COVID-19 Vaccination among People Living with HIV and AIDS in China: Nationwide Cross-sectional Online Survey. JMIR Public Health Surveill. 2021, 7, e31125. [CrossRef] [PubMed]

29. Zhang, Y.; Zeng, G.; Pan, H.; Li, C.; Hu, Y.; Chu, K.; Han, W.X.; Chen, Z.; Tang, R.; Yin, W.D.; et al. Safety, tolerability, and immunogenicity of an inactivated SARS-CoV-2 vaccine in healthy adults aged 18-59 years: A randomized, double-blind, placebo-controlled, phase 1/2 clinical trial. Lancet Infect. Dis. 2021, 21, 181-192. [CrossRef]

30. National Center for AIDS/STD Control and Prevention CC. National Guideline for Detection of HIV / AIDS. Available online: http:/ / ncaids.chinacdc.cn/zxzx/zxdteff/202005/t20200518_216798.htm (accessed on 5 August 2021).

31. Catherine, F.-X.; Piroth, L. Hepatitis B virus vaccination in HIV-infected people: A review. Hum. Vaccines Immunother. 2017, 13, 1-10. [CrossRef] [PubMed]

32. Hoffman, S.A.; Costales, C.; Sahoo, M.K.; Palanisamy, S.; Yamamoto, F.; Huang, C.; Verghese, M.; Solis, D.A.; Sibai, M.; Subramanian, A.; et al. SARS-CoV-2 Neutralization Resistance Mutations in Patient with HIV/AIDS, California, USA. Emerg. Infect. Dis. 2021, 27, 2720. [CrossRef] [PubMed] 
33. Jelicic, K.; Cimbro, R.; Nawaz, F.; Huang da, W.; Zheng, X.; Yang, J.; Lempicki, R.A.; Pascuccio, M.; Van Ryk, D.; Schwing, C.; et al. The HIV-1 envelope protein gp120 impairs B cell proliferation by inducing TGF- $\beta 1$ production and FcRL4 expression. Nat. Immunol. 2013, 14, 1256-1265. [CrossRef] [PubMed]

34. Okoye, A.A.; Picker, L.J. CD4+T-cell depletion in HIV infection: Mechanisms of immunological failure. Immunol. Rev. 2013, 254, 54-64. [CrossRef] [PubMed]

35. Cao, Y.; Su, B.; Guo, X.; Sun, W.; Deng, Y.; Bao, L.; Zhu, Q.Y.; Zhang, X.; Zheng, Y.H.; Geng, C.Y.; et al. Potent Neutralizing Antibodies against SARS-CoV-2 Identified by High-Throughput Single-Cell Sequencing of Convalescent Patients' B Cells. Cell 2020, 182, 73-84.e16. [CrossRef] [PubMed]

36. Klein, S.L.; Flanagan, K.L. Sex differences in immune responses. Nat. Rev. Immunol. 2016, 16, 626-638. [CrossRef] [PubMed] 